\title{
ON A NEW CLASS OF STRUCTURED MATRICES RELATED TO THE DISCRETE SKEW-SELF-ADJOINT DIRAC SYSTEMS*
}

\author{
B. FRITZSCHE ${ }^{\dagger}$, B. KIRSTEIN ${ }^{\dagger}$, AND A.L. SAKHNOVICH $^{\ddagger}$
}

\begin{abstract}
A new class of the structured matrices related to the discrete skew-self-adjoint Dirac systems is introduced. The corresponding matrix identities and inversion procedure are treated. Analogs of the Schur coefficients and of the Christoffel-Darboux formula are studied. It is shown that the structured matrices from this class are always positive-definite, and applications for an inverse problem for the discrete skew-self-adjoint Dirac system are obtained.
\end{abstract}

Key words. Structured matrices, Matrix identity, Schur coefficients, Christoffel-Darboux formula, Transfer matrix function, Discrete skew-self-adjoint Dirac system, Weyl function, Inverse problem.

AMS subject classifications. 15A09, 15A24, 39A12.

1. Introduction. It is well-known that Toeplitz and block Toeplitz matrices are closely related to a discrete system of equations, namely to Szegö recurrence. This connection have been actively studied during the last decades. See, for instance, [1]$[5],[12,25]$ and numerous references therein. The connections between block Toeplitz matrices and Weyl theory for the self-adjoint discrete Dirac system were treated in [11]. (See [26] for the Weyl theory of the discrete analog of the Schrödinger equation.) The Weyl theory for the skew-self-adjoint discrete Dirac system

$$
W_{k+1}(\lambda)-W_{k}(\lambda)=-\frac{i}{\lambda} C_{k} W_{k}(\lambda), \quad C_{k}=C_{k}^{*}=C_{k}^{-1}, \quad k=0,1, \ldots
$$

was developed in $[14,18]$. Here $C_{k}$ are $2 p \times 2 p$ matrix functions. When $p=1$, system (1.1) is an auxiliary linear system for the isotropic Heisenberg magnet model. Explicit solutions of the inverse problem were constructed in [14]. A general procedure to construct the solutions of the inverse problem for system (1.1) was given in [18], using a new class of structured matrices $S$, which satisfy the matrix identity

$$
A S-S A^{*}=i \Pi \Pi^{*} .
$$

*Received by the editors May 19, 2008. Accepted for publication September 11, 2008. Handling Editor: Harm Bart.

†Fakultät für Mathematik und Informatik, Mathematisches Institut, Universität Leipzig, Johannisgasse 26, D-04103 Leipzig, Germany (fritzsche@math.uni-leipzig.de, kirstein@math.uni-leipzig.de).

${ }^{\ddagger}$ Fakultät für Mathematik, Universität Wien, Nordbergstrasse 15, A-1090 Wien, Austria (al_sakhnov@yahoo.com). Supported by the Austrian Science Fund (FWF) under Grant no. Y330. 
Here, $S$ and $A$ are $(n+1) p \times(n+1) p$ matrices and $\Pi$ is an $(n+1) p \times 2 p$ matrix. The block matrix $A$ has the form

$$
A:=A(n)=\left\{a_{j-k}\right\}_{k, j=0}^{n}, \quad a_{r}=\left\{\begin{array}{cll}
0 & \text { for } \quad r>0 \\
\frac{i}{2} I_{p} & \text { for } \quad r=0 \\
i I_{p} & \text { for } \quad r<0
\end{array},\right.
$$

where $I_{p}$ is the $p \times p$ identity matrix. The matrix $\Pi=\left[\begin{array}{ll}\Phi_{1} & \Phi_{2}\end{array}\right]$ consists of two block columns of the form

$$
\Phi_{1}=\left[\begin{array}{c}
I_{p} \\
I_{p} \\
\vdots \\
I_{p}
\end{array}\right], \quad \Phi_{2}=\left[\begin{array}{c}
\alpha_{0} \\
\alpha_{0}+\alpha_{1} \\
\vdots \\
\alpha_{0}+\alpha_{1}+\cdots+\alpha_{n}
\end{array}\right] .
$$

DEFINITION 1.1. The class of the block matrices $S$ determined by the matrix identity (1.2) and formulas (1.3) and (1.4) is denoted by $\Omega_{n}$.

Notice that the blocks $\alpha_{k}$ in [18] are Taylor coefficients of the Weyl functions and that the matrices $C_{n}(0 \leq n \leq l)$ in (1.1) are easily recovered from the expressions $\Pi(n)^{*} S(n)^{-1} \Pi(n)(0 \leq n \leq l)$ (see Theorem 3.4 of [18]). In this way, the structure of the matrices $S$ determined by the matrix identity (1.2) and formulas (1.3) and (1.4), their inversion and conditions of invertibility prove essential. Recall that the selfadjoint block Toeplitz matrices satisfy [15]-[17] the identity $A S-S A^{*}=i \Pi J \Pi^{*}(J=$ $\left.\left[\begin{array}{cc}0 & I_{p} \\ I_{p} & 0\end{array}\right]\right)$, which is close to $(1.2)-(1.4)$. We refer also to $[20]-[24]$ and references therein for the general method of the operator identities. The analogs of various results on the Toeplitz matrices and $j$-theory from [6]-[11] can be obtained for the class $\Omega_{n}$, too.

2. Structure of the matrices from $\Omega_{n}$. Consider first the block matrix $S=$ $\left\{s_{k j}\right\}_{k, j=0}^{n}$ with the $p \times p$ entries $s_{k j}$, which satisfies the identity

$$
A S-S A^{*}=i Q, \quad Q=\left\{q_{k j}\right\}_{k, j=0}^{n} .
$$

One can easily see that the equality

$$
q_{k j}=s_{k j}+\sum_{r=0}^{k-1} s_{r j}+\sum_{r=0}^{j-1} s_{k r}
$$

follows from (2.1). Sometimes we add comma between the indices and write $s_{k, j}$. Putting $s_{-1, j}=s_{k,-1}=q_{-1, j}=q_{k,-1}=0$, from (2.2) we have

$$
s_{k+1, j+1}-s_{k j}=q_{k j}+q_{k+1, j+1}-q_{k+1, j}-q_{k, j+1}, \quad-1 \leq k, j \leq n-1 .
$$


Now, putting $Q=i \Pi \Pi^{*}$ and taking into account (2.3), we get the structure of $S$.

Proposition 2.1. Let $S \in \Omega_{n}$. Then we have

$$
s_{k+1, j+1}-s_{k j}=\alpha_{k+1} \alpha_{j+1}^{*} \quad(-1 \leq k, j \leq n-1),
$$

excluding the case when $k=-1$ and $j=-1$ simultaneously. For that case, we have

$$
s_{00}=I_{p}+\alpha_{0} \alpha_{0}^{*} .
$$

Notice that for the block Toeplitz matrix, the equalities $s_{k+1, j+1}-s_{k j}=0(0 \leq$ $k, j \leq n-1)$ hold. Therefore, Toeplitz and block Toeplitz matrices can be used to study certain homogeneous processes and appear as a result of discretization of homogeneous equations. From this point of view, the matrix $S \in \Omega_{n}$ is perturbed by the simplest inhomogeneity.

The authors are grateful to the referee for the next interesting remark.

REMARK 2.2. From (1.2)-(1.4) we get another useful identity, namely,

$$
S-N S N^{*}=\widehat{\Pi} \widehat{\Pi}^{*},
$$

where

$$
N=\left\{\delta_{k-j-1} I_{p}\right\}_{k, j=0}^{n}=\left[\begin{array}{cccc}
0 & & & 0 \\
I_{p} & & & 0 \\
& \ddots & & \vdots \\
& & I_{p} & 0
\end{array}\right], \quad \widehat{\Pi}=\left[\begin{array}{cc}
I_{p} & \alpha_{0} \\
0 & \alpha_{1} \\
\vdots & \vdots \\
0 & \alpha_{n}
\end{array}\right] .
$$

Indeed, it is easy to see that $\left(I_{(n+1) p}-N\right) A=\frac{i}{2}\left(I_{(n+1) p}+N\right)$. Hence, the identity

$$
i\left(S-N S N^{*}\right)=i\left(I_{(n+1) p}-N\right) \Pi \Pi^{*}\left(I_{(n+1) p}-N^{*}\right)
$$

follows from (1.2). By (2.7), we have $\left(I_{(n+1) p}-N\right) \Pi=\widehat{\Pi}$, and so (2.6) is valid. Relations (2.4) and (2.5) are immediate from (2.6).

Proposition 2.3. Let $S=\left\{s_{k j}\right\}_{k, j=0}^{n} \in \Omega_{n}$. Then $S$ is positive and, moreover, $S \geq I_{(n+1) p}$. We have $S>I_{(n+1) p}$ if and only if $\operatorname{det} \alpha_{0} \neq 0$.

Proof. From (2.5) it follows that $S(0)=s_{00} \geq I_{p}$ and that $S(0)>I_{p}$, when $\operatorname{det} \alpha_{0} \neq 0$. The necessity of $\operatorname{det} \alpha_{0} \neq 0$, for the inequality $S>I_{(n+1) p}$ to be true, follows from (2.5), too. We shall prove that $S \geq I_{(n+1) p}$ and that $S>I_{(n+1) p}$, when $\operatorname{det} \alpha_{0} \neq 0$, by induction.

Suppose that $S(r-1)=\left\{s_{k j}\right\}_{k, j=0}^{r-1} \geq I_{r p}(r \geq 1)$. According to (2.6), we can 
present $S(r)=\left\{s_{k j}\right\}_{k, j=0}^{r}$ in the form $S(r)=S_{1}+S_{2}$,

$$
S_{1}:=\left[\begin{array}{c}
\alpha_{0} \\
\alpha_{1} \\
\vdots \\
\alpha_{r}
\end{array}\right]\left[\begin{array}{llll}
\alpha_{0}^{*} & \alpha_{1}^{*} & \cdots & \alpha_{r}^{*}
\end{array}\right], \quad S_{2}:=\left[\begin{array}{cc}
I_{p} & 0 \\
0 & S(r-1)
\end{array}\right] .
$$

By the assumption of induction, it is immediate that $S(r) \geq S_{2} \geq I_{(r+1) p}$. Hence, we get $S=S(n) \geq I_{(n+1) p}$.

Suppose that $\operatorname{det} \alpha_{0} \neq 0$ and $S(r-1)>I_{(n+1) p}$. Let $S(r) f=f\left(f \in B C^{(r+1) p}\right)$, i.e., let $f^{*}\left(S(r)-I_{(r+1) p}\right) f=0$. By (2.8), we have $S_{1} \geq 0$, and by the assumption of induction, we have $S_{2}-I_{(r+1) p} \geq 0$. So, it follows from $f^{*}\left(S(r)-I_{(r+1) p}\right) f=0$ that $f^{*} S_{1} f=0$ and $f^{*}\left(S_{2}-I_{(r+1) p}\right) f=0$. Hence, as $\alpha_{0} \alpha_{0}^{*}>0$ and $S(r-1)>I_{r p}$, we derive $f=0$. In other words, $S(r) f=f$ implies $f=0$, that is, $\operatorname{det}\left(S(r)-I_{(r+1) p}\right) \neq 0$. From $\operatorname{det}\left(S(r)-I_{(r+1) p}\right) \neq 0$ and $S(r) \geq I_{(r+1) p}$, we get $S(r)>0$. So, the condition $\operatorname{det} \alpha_{0} \neq 0$ implies $S(n)>I_{(n+1) p}$ by induction.

REMARK 2.4. Using formula (2.5) and representations $S(r)=S_{1}(r)+S_{2}(r)$ $(0<r \leq n)$, where $S_{1}(r)$ and $S_{2}(r)$ are given by (2.8), one easily gets

$$
\begin{aligned}
& S=I_{(n+1) p}+\left[\begin{array}{c}
\alpha_{0} \\
\alpha_{1} \\
\vdots \\
\alpha_{n}
\end{array}\right]\left[\begin{array}{llll}
\alpha_{0}^{*} & \alpha_{1}^{*} & \cdots & \alpha_{n}^{*}
\end{array}\right] \\
& +\left[\begin{array}{c}
0 \\
\alpha_{0} \\
\vdots \\
\alpha_{n-1}
\end{array}\right]\left[\begin{array}{llll}
0 & \alpha_{0}^{*} & \cdots & \alpha_{n-1}^{*}
\end{array}\right]+\cdots+\left[\begin{array}{c}
0 \\
\vdots \\
0 \\
\alpha_{0}
\end{array}\right]\left[\begin{array}{llll}
0 & \cdots & 0 & \alpha_{0}^{*}
\end{array}\right] \\
& =I_{(n+1) p}+V_{\alpha} V_{\alpha}^{*}, \quad V_{\alpha}:=\left[\begin{array}{ccccc}
\alpha_{0} & 0 & 0 & \cdots & 0 \\
\alpha_{1} & \alpha_{0} & 0 & \cdots & 0 \\
\vdots & \vdots & \vdots & & \vdots \\
\alpha_{n} & \alpha_{n-1} & \alpha_{n-2} & \cdots & \alpha_{0}
\end{array}\right] \text {. }
\end{aligned}
$$

Here, $V_{\alpha}$ is a triangular block Toeplitz matrix, and formula (2.9) is another way to prove Proposition 2.3. Further, we will be interested in a block triangular factorization of the matrix $S$ itself, namely, $S=V_{-}^{-1}\left(V_{-}^{*}\right)^{-1}$, where $V_{-}$is a lower triangular matrix.

Similar to the block Toeplitz case (see [13] and references therein) the matrices $S \in \Omega_{n}$ admit the matrix identity of the form $A_{1} S-S A_{1}=Q_{1}$, where $Q_{1}$ is of low 
rank, $A_{1}:=\left\{\delta_{k-j+1} I_{p}\right\}_{k, j=0}^{n}=N^{*}$ and $N$ is given in (2.7). The next proposition follows easily from (2.4).

Proposition 2.5. Let $S \in \Omega_{n}$. Then we have

(2.10) $A_{1} S-S A_{1}=y_{1} y_{2}^{*}+y_{3} y_{4}^{*}+y_{5} y_{6}^{*}, A_{1}^{*} S-S A_{1}^{*}=-\left(y_{2} y_{1}^{*}+y_{4} y_{3}^{*}+y_{6} y_{5}^{*}\right)$,

where

$$
y_{1}=\left[\begin{array}{c}
s_{10} \\
s_{20} \\
\vdots \\
s_{n 0} \\
0
\end{array}\right], \quad y_{3}=-\left[\begin{array}{c}
0 \\
0 \\
\vdots \\
0 \\
I_{p}
\end{array}\right], \quad y_{5}=\left[\begin{array}{c}
\alpha_{1} \\
\alpha_{2} \\
\vdots \\
\alpha_{n} \\
0
\end{array}\right], \quad y_{6}=\left[\begin{array}{c}
0 \\
\alpha_{1} \\
\alpha_{2} \\
\vdots \\
\alpha_{n}
\end{array}\right]
$$

$$
y_{2}^{*}=\left[\begin{array}{lllll}
I_{p} & 0 & 0 & \cdots & 0
\end{array}\right], \quad y_{4}^{*}=\left[\begin{array}{lllll}
0 & s_{n 0} & s_{n 1} & \cdots & s_{n, n-1}
\end{array}\right] .
$$

Differently than the block Toeplitz matrix case, the rank of $A_{1} S-S A_{1}$ is in general situation larger than the rank of $A S-S A^{*}$, where $A$ is given by (1.3). (To see this compare (1.2)-(1.4) and (2.10)-(2.12).)

3. Transfer matrix function and Weyl functions. Introduce the $(r+1) p \times$ $(n+1) p$ matrix

$$
P_{k}:=\left[\begin{array}{ll}
I_{(r+1) p} & 0
\end{array}\right], \quad r \leq n .
$$

It follows from (1.3) that $P_{r} A(n)=A(r) P_{r}$. Hence, using (1.2) we derive

$$
A(r) S(r)-S(r) A(r)^{*}=i \Pi(r) \Pi(r)^{*}, \quad \Pi(r):=P_{r} \Pi .
$$

As $S>0$, it admits a block triangular factorization

$$
S=V_{-}^{-1}\left(V_{-}^{*}\right)^{-1},
$$

where $V_{-}^{ \pm 1}$ are block lower triangular matrices. It is immediate from (3.3) that

$$
S(r)=V_{-}(r)^{-1}\left(V_{-}(r)^{*}\right)^{-1}, \quad V_{-}(r):=P_{r} V_{-} P_{r}^{*} .
$$

Recall that $S$-node $[21,23,24]$ is the triple $(A(r), S(r), \Pi(r))$ that satisfies the matrix identity (3.2) (see also $[21,23,24]$ for a more general definition of the $S$ node). Following $[21,23,24]$, introduce the transfer matrix function corresponding to the $S$-node:

$$
w_{A}(r, \lambda)=I_{2 p}-i \Pi(r)^{*} S(r)^{-1}\left(A(r)-\lambda I_{(r+1) p}\right)^{-1} \Pi(r)
$$


In particular, taking into account (3.4) and (3.5), we get

$$
w_{A}(0, \lambda)=I_{2 p}-\frac{2 i}{i-2 \lambda} \beta(0)^{*} \beta(0), \quad \beta(0)=V_{-}(0) \Pi(0) .
$$

By the factorization theorem 4 from [21] (see also [23, p. 188]), we have

$$
\begin{aligned}
w_{A}(r, \lambda)=\left(I_{2 p}-\right. & i \Pi(r)^{*} S(r)^{-1} P^{*}\left(P A(r) P^{*}-\lambda I_{p}\right)^{-1}\left(P S(r)^{-1} P^{*}\right)^{-1} \\
& \left.\times P S(r)^{-1} \Pi(r)\right) w_{A}(r-1, \lambda), \quad P=\left[\begin{array}{lllll}
0 & \cdots & 0 & I_{p}
\end{array}\right] .
\end{aligned}
$$

According to (1.3), we obtain

$$
\left(P A(r) P^{*}-\lambda I_{p}\right)^{-1}=\left(\frac{i}{2}-\lambda\right)^{-1} I_{p}
$$

Using (3.4), we derive

$$
P S(r)^{-1} P^{*}=\left(V_{-}(r)\right)_{r r}^{*}\left(V_{-}(r)\right)_{r r}, \quad P S(r)^{-1} \Pi(r)=\left(V_{-}(r)\right)_{r r}^{*} P V_{-}(r) \Pi(r),
$$

where $\left(V_{-}(r)\right)_{r r}$ is the block entry of $V_{-}(r)$ (the entry from the $r$-th block row and the $r$-th block column). In view of (3.8) and (3.9), we rewrite (3.7) in the form

$$
\begin{array}{r}
w_{A}(r, \lambda)=\left(I_{2 p}-\frac{2 i}{i-2 \lambda} \beta(r)^{*} \beta(r)\right) w_{A}(r-1, \lambda), \\
\beta(r)=P V_{-}(r) \Pi(r)=\left(V_{-} \Pi\right)_{r}, \quad 0<r \leq n .
\end{array}
$$

Here, $\left(V_{-} \Pi\right)_{r}$ is the $r$-th $p \times 2 p$ block of the block column vector $V_{-} \Pi$. Moreover, according to (3.9) and definitions (3.6), (3.11) of $\beta$, we have

$$
\begin{aligned}
& \left(P S(r)^{-1} P^{*}\right)^{-\frac{1}{2}} P S(r)^{-1} \Pi(r)=u(r) \beta(r), \\
& u(r):=\left(P S(r)^{-1} P^{*}\right)^{-\frac{1}{2}}\left(V_{-}(r)\right)_{r r}^{*}, \quad u(r)^{*} u(r)=I_{p} .
\end{aligned}
$$

As $u$ is unitary, the properties of $\left(P S(r)^{-1} P^{*}\right)^{-\frac{1}{2}} P S(r)^{-1} \Pi(r)$ proved in [18, p. 2098] imply the next proposition.

Proposition 3.1. Let $S \in \Omega_{n}$ and let $\beta(k)(0 \leq k \leq n)$ be given by (3.3), (3.4), (3.6) and (3.11). Then we have

$$
\left\{\begin{array}{l}
\beta(k) \beta(k)^{*}=I_{p} \quad(0 \leq k \leq n), \\
\operatorname{det} \beta(k-1) \beta(k)^{*} \neq 0 \quad(0<k \leq n), \\
\operatorname{det} \beta_{1}(0) \neq 0,
\end{array}\right.
$$

where $\beta_{1}(k), \beta_{2}(k)$ are $p \times p$ blocks of $\beta(k)$. 
REMARK 3.2. Notice that the lower triangular factor $V_{-}$is not defined by $S$ uniquely. Hence, the matrices $\beta(k)$ are not defined uniquely, too. Nevertheless, in view of (3.12), the matrices $\beta(k)^{*} \beta(k)$ are uniquely defined, which suffices for our considerations.

When $p=1$ and $C_{k} \neq \pm I_{2}$, the matrices $C_{k}=C_{k}^{*}=C_{k}^{-1}$ (i.e., the potential of the system (1.1)) can be presented in the form $C_{k}=I_{2}-2 \beta(k)^{*} \beta(k)$, where $\beta(k) \beta(k)^{*}=1$. Therefore, it is assumed in [18] for the system (1.1) on the interval $0 \leq k \leq n$, that

$$
C_{k}=I_{2 p}-2 \beta(k)^{*} \beta(k),
$$

where $\beta(k)$ are $p \times 2 p$ matrices and (3.13) holds. Relation (3.14) implies $C_{k}=U_{k} j U_{k}^{*}$, where $j=\left[\begin{array}{cc}-I_{p} & 0 \\ 0 & I_{p}\end{array}\right]$ and $U_{k}$ are unitary $2 p \times 2 p$ matrices. The equalities $C_{k}=$ $C_{k}^{*}=C_{k}^{-1}$ follow. Consider the fundamental solution $W_{r}(\lambda)$ of the system (1.1) normalized by $W_{0}(\lambda)=I_{2 p}$. Using (3.6) and (3.10), one easily derives

$$
W_{r+1}(\lambda)=\left(\frac{\lambda-i}{\lambda}\right)^{r+1} w_{A}\left(r, \frac{\lambda}{2}\right), \quad 0 \leq r \leq n .
$$

Similar to the continuous case, the Weyl functions of the system (1.1) are defined via Möbius (linear-fractional) transformation

$$
\varphi(\lambda)=\left(\mathcal{W}_{11}(\lambda) R(\lambda)+\mathcal{W}_{12}(\lambda) Q(\lambda)\right)\left(\mathcal{W}_{21}(\lambda) R(\lambda)+\mathcal{W}_{22}(\lambda) Q(\lambda)\right)^{-1},
$$

where $\mathcal{W}_{i j}$ are $p \times p$ blocks of $\mathcal{W}$ and

$$
\mathcal{W}(\lambda)=\left\{\mathcal{W}_{i j}(\lambda)\right\}_{i, j=1}^{2}:=W_{n+1}(\bar{\lambda})^{*}
$$

Here, $R$ and $Q$ are any $p \times p$ matrix functions analytic in the neighborhood of $\lambda=i$ and such that

$$
\operatorname{det}\left(\mathcal{W}_{21}(i) R(i)+\mathcal{W}_{22}(i) Q(i)\right) \neq 0
$$

One can easily verify that such pairs always exist (see [18, p. 2090]). A matrix function $\varphi(\lambda)$ of order $p$, analytic at $\lambda=i$, generates a matrix $S \in \Omega_{n}$ via the Taylor coefficients

$$
\varphi\left(i \frac{1+z}{1-z}\right)=-\left(\alpha_{0}+\alpha_{1} z+\cdots+\alpha_{n} z^{n}\right)+O\left(z^{n+1}\right) \quad(z \rightarrow 0)
$$

and identity (1.2). By Theorem 3.7 in [18], such $\varphi$ is a Weyl function of some system (1.1) if and only if $S$ is invertible. Now, from Proposition 2.3 it follows that $S>0$, and the next proposition is immediate. 
Proposition 3.3. Any $p \times p$ matrix function $\varphi$, which is analytic at $\lambda=i$, is a Weyl function of some system (1.1) on the interval $0 \leq k \leq n$, such that (3.13) and (3.14) hold.

Moreover, from the proof of the statement (ii) of Theorem 3.7 in [18], the Corollary 3.6 in [18] and our Proposition 3.3, we get:

Proposition 3.4. Let the $p \times p$ matrix function $\varphi$ be analytic at $\lambda=i$ and admit expansion (3.19). Then $\varphi$ is a Weyl function of the system (1.1) $(0 \leq k \leq n)$, where $C_{k}$ are defined by the formulas (1.2)-(1.4), $\Pi=\left[\begin{array}{ll}\Phi_{1} & \Phi_{2}\end{array}\right]$, (3.3), (3.11) and (3.14). Moreover, any Weyl function of this system admits expansion (3.19).

4. Schur coefficients and Christoffel-Darboux formula. The sequence $\left\{\alpha_{k}\right\}_{k=0}^{n}$ uniquely determines via formulas (1.2)-(1.4) or (1.3), (1.4), (2.4) and (2.5) the $S$-node $(A, S, \Pi)$. Then, using (3.3), (3.11) and (3.14), we uniquely recover the system (1.1) $(0 \leq k \leq n)$, or equivalently, we recover the sequence $\left\{\beta_{k}^{*} \beta_{k}\right\}_{k=0}^{n}$, such that (3.13) holds. By Proposition 3.4, one can use Weyl functions of this system to obtain the sequence $\left\{\alpha_{k}\right\}_{k=0}^{n}$.

REMARK 4.1. Thus, there are one to one correspondences between the sequences $\left\{\alpha_{k}\right\}_{k=0}^{n}$, the $S$-nodes $(A, S, \Pi)$ satisfying (1.2), the systems (1.1) $(0 \leq k \leq n)$ with $C_{k}$ of the form (3.14) and the sequences $\left\{\beta_{k}^{*} \beta_{k}\right\}_{k=0}^{n}$, such that (3.13) holds.

Next, we consider a correspondence between $\left\{\beta_{k}^{*} \beta_{k}\right\}_{k=0}^{n}$ and some $p \times p$ matrices $\left\{\rho_{k}\right\}_{k=0}^{n}\left(\left\|\rho_{k}\right\| \leq 1\right)$. Notice that $0 \leq \beta_{1}(k) \beta_{1}(k)^{*} \leq I_{p}$, and suppose that these inequalities are strict:

$$
0<\beta_{1}(k) \beta_{1}(k)^{*}<I_{p} \quad(0 \leq k \leq n) .
$$

In view of the first relation in (3.13) and inequalities (4.1), we have $\operatorname{det} \beta_{1}(k) \neq 0$ and $\operatorname{det} \beta_{2}(k) \neq 0$. So, we can put

$$
\rho_{k}:=\left(\beta_{2}(k)^{*} \beta_{2}(k)\right)^{-\frac{1}{2}} \beta_{2}(k)^{*} \beta_{1}(k) .
$$

It follows from (4.2) that

$$
\begin{aligned}
\rho_{k} \rho_{k}^{*} & =\left(\beta_{2}(k)^{*} \beta_{2}(k)\right)^{-\frac{1}{2}} \beta_{2}(k)^{*}\left(I_{p}-\beta_{2}(k) \beta_{2}(k)^{*}\right) \beta_{2}(k)\left(\beta_{2}(k)^{*} \beta_{2}(k)\right)^{-\frac{1}{2}} \\
& =I_{p}-\beta_{2}(k)^{*} \beta_{2}(k) .
\end{aligned}
$$

By (4.2) and (4.3), we obtain

$$
\left[\rho_{k} \quad\left(I_{p}-\rho_{k} \rho_{k}^{*}\right)^{\frac{1}{2}}\right]=u_{k} \beta(k), \quad\left\|\rho_{k}\right\|<1,
$$

where

$$
u_{k}:=\left(\beta_{2}(k)^{*} \beta_{2}(k)\right)^{-\frac{1}{2}} \beta_{2}(k)^{*}, \quad u_{k} u_{k}^{*}=I_{p},
$$


i.e., $u_{k}$ is unitary.

REMARK 4.2. Under condition (4.1), according to (4.4) and (4.5), the sequence $\left\{\beta_{k}^{*} \beta_{k}\right\}_{k=0}^{n}$ is uniquely recovered from the sequence $\left\{\rho_{k}\right\}_{k=0}^{n}\left(\left\|\rho_{k}\right\| \leq 1\right)$ :

$$
\beta_{k}^{*} \beta_{k}=\left[\begin{array}{c}
\rho_{k}^{*} \\
\left(I_{p}-\rho_{k} \rho_{k}^{*}\right)^{\frac{1}{2}}
\end{array}\right]\left[\begin{array}{ll}
\rho_{k} & \left(I_{p}-\rho_{k} \rho_{k}^{*}\right)^{\frac{1}{2}}
\end{array}\right] .
$$

By Remark 4.1 this means that the $S$-node can be recovered from the sequence $\left\{\rho_{k}\right\}_{k=0}^{n}$. Therefore, similar to the Toeplitz case, we call $\rho_{k}$ the Schur coefficients of the $S$-node $(A, S, \Pi)$.

Besides Schur coefficients, we obtain an analog of the Christoffel-Darboux formula.

Proposition 4.3. Let $S \in \Omega_{n}$, let $w_{A}(r, \lambda)$ be introduced by (3.5) for $r \geq 0$ and put $w_{A}(-1, \lambda)=I_{2 p}$. Then we have

$$
\begin{aligned}
& \sum_{k=-1}^{n-1} w_{A}(k, \mu)^{*} \beta(k+1)^{*} \beta(k+1) w_{A}(k, \lambda) \\
& \quad=\frac{(2 \lambda-i)(2 \bar{\mu}+i)}{4 i(\bar{\mu}-\lambda)}\left(w_{A}(n, \mu)^{*} w_{A}(n, \lambda)-I_{2 p}\right) .
\end{aligned}
$$

Proof. From (3.10) it follows that

$$
\begin{aligned}
& w_{A}(k+1, \mu)^{*} w_{A}(k+1, \lambda)-w_{A}(k, \mu)^{*} w_{A}(k, \lambda)= \\
& w_{A}(k, \mu)^{*}\left(\left(I_{2 p}-\frac{2 i}{2 \bar{\mu}+i} \beta(k+1)^{*} \beta(k+1)\right)\right. \\
& \left.\times\left(I_{2 p}+\frac{2 i}{2 \lambda-i} \beta(k+1)^{*} \beta(k+1)\right)-I_{2 p}\right) w_{A}(k, \lambda) .
\end{aligned}
$$

Using $\beta(k) \beta(k)^{*}=I_{p}$, we rewrite (4.8) in the form

$$
\begin{aligned}
& w_{A}(k+1, \mu)^{*} w_{A}(k+1, \lambda)-w_{A}(k, \mu)^{*} w_{A}(k, \lambda) \\
& =\frac{4 i(\bar{\mu}-\lambda)}{(2 \lambda-i)(2 \bar{\mu}+i)} w_{A}(k, \mu)^{*} \beta(k+1)^{*} \beta(k+1) w_{A}(k, \lambda) .
\end{aligned}
$$

Equality (4.7) follows from (4.9).

5. Inversion of $S \in \Omega_{n}$. To recover the system (1.1) from $\left\{\alpha_{k}\right\}_{k=0}^{n}$, it is convenient to use formula (3.11). The matrices $V_{-}(r)(r \geq 0)$ in this formula can be constructed recursively. 
Proposition 5.1. Let $S=V_{-}^{-1}\left(V_{-}^{*}\right)^{-1} \in \Omega_{n}$. Then $V_{-}(r+1)(0 \leq r<n)$ can be constructed by the formula

$$
V_{-}(r+1)=\left[\begin{array}{cc}
V_{-}(r) & 0 \\
-t(r) S_{21}(r) V_{-}(r)^{*} V_{-}(r) & t(r)
\end{array}\right],
$$

where $S_{21}(r)=\left[\begin{array}{llll}s_{r+1,0} & s_{r+1,1} & \ldots & s_{r+1, r}\end{array}\right]$

$$
t(r)=\left(s_{r+1, r+1}-S_{21}(r) V_{-}(r)^{*} V_{-}(r) S_{21}(r)^{*}\right)^{-\frac{1}{2}} .
$$

Proof. To prove the proposition it suffices to assume that $V_{-}(r)$ satisfies (3.4) and prove $S(r+1)=V_{-}(r+1)^{-1}\left(V_{-}(r+1)^{*}\right)^{-1}$. In view of Proposition 2.3 and (3.4), we have $s_{r+1, r+1}-S_{21}(r) V_{-}(r)^{*} V_{-}(r) S_{21}(r)^{*}>0$, i.e., formula (5.2) is well defined. Now, it is easily checked that $S(r+1)^{-1}=V_{-}(r+1)^{*} V_{-}(r+1)$ (see formula (2.7) in $[17])$.

Put $T=\left\{t_{k j}\right\}_{k, j=0}^{n}=S^{-1}$,

$$
\widehat{Q}=\left\{\widehat{q}_{k j}\right\}_{k, j=0}^{n}=T \Pi \Pi^{*} T, \quad X=T \Phi_{1}, \quad Y=T \Phi_{2},
$$

where $t_{k j}$ and $\widehat{q}_{k j}$ are $p \times p$ blocks of $T$ and $\widehat{Q}$, respectively. Similar to $[15,16,20,22]$ and references therein, we get the next proposition.

Proposition 5.2. Let $S \in \Omega_{n}$. Then $T=S^{-1}$ is recovered from $X$ and $Y$ by the formula

$$
t_{k j}=\widehat{q}_{k j}+\widehat{q}_{k+1, j+1}-\widehat{q}_{k+1, j}-\widehat{q}_{k, j+1}+t_{k+1, j+1},
$$

or, equivalently, by the formula

$$
t_{k j}=\widehat{q}_{k j}+2 \sum_{r=1}^{n-k} \widehat{q}_{k+r, j+r}-\sum_{r=1}^{n-k} \widehat{q}_{k+r, j+r-1}-\sum_{r=1}^{n-k+1} \widehat{q}_{k+r-1, j+r},
$$

where we fix $t_{k j}=0$ and $\widehat{q}_{k j}=0$ for $k>n$ or $j>n$, and

$$
\widehat{Q}=X X^{*}+Y Y^{*} .
$$

The block vectors $X$ and $Y$ are connected by the relations

$$
\begin{aligned}
\sum_{r=0}^{n}\left(X_{r}-X_{r}^{*}\right)=0, & \sum_{r=0}^{n-k} X_{n-r}=\sum_{r=0}^{n-k} \widehat{q}_{k+r, r} \quad(k \geq 0), \\
\sum_{r=0}^{n-k} X_{n-r}^{*} & =\sum_{r=0}^{n-k} \widehat{q}_{r, k+r} \quad(k>0) .
\end{aligned}
$$


Proof. From the identity (1.2) and formula (5.3), it follows that

$$
T A-A^{*} T=i \widehat{Q},
$$

where $\widehat{Q}$ satisfies (5.6). The identity $T A-A^{*} T=i \widehat{Q}$ yields (5.4), which, in its turn, implies (5.5).

To derive (5.7), we rewrite (5.8) in the form

$$
\begin{aligned}
& \left(A^{*}-\lambda I_{(n+1) p}\right)^{-1} T-T\left(A-\lambda I_{(n+1) p}\right)^{-1} \\
& =i\left(A^{*}-\lambda I_{(n+1) p}\right)^{-1} \widehat{Q}\left(A-\lambda I_{(n+1) p}\right)^{-1},
\end{aligned}
$$

and multiply both sides of (5.9) by $\Phi_{1}$ from the right and by $\Phi_{1}^{*}$ from the left. Taking into account (5.3), we get

$$
\begin{gathered}
\Phi_{1}^{*}\left(A^{*}-\lambda I_{(n+1) p}\right)^{-1} X-X^{*}\left(A-\lambda I_{(n+1) p}\right)^{-1} \Phi_{1} \\
\quad=i \Phi_{1}^{*}\left(A^{*}-\lambda I_{(n+1) p}\right)^{-1} \widehat{Q}\left(A-\lambda I_{(n+1) p}\right)^{-1} \Phi_{1} .
\end{gathered}
$$

It is easily checked (see formula (1.10) in [17]) that

$$
\begin{array}{r}
\left(A-\lambda I_{(n+1) p}\right)^{-1} \Phi_{1}=\left(\frac{i}{2}-\lambda\right)^{-1} \operatorname{col}\left[\begin{array}{lllll}
I_{p} & \zeta^{-1} I_{p} & \cdots & \zeta^{-n} & I_{p}
\end{array}\right] \\
\Phi_{1}^{*}\left(A^{*}-\lambda I_{(n+1) p}\right)^{-1}=-\left(\frac{i}{2}+\lambda\right)^{-1}\left[\begin{array}{lllll}
I_{p} & \zeta I_{p} & \cdots & \zeta^{n} I_{p}
\end{array}\right],
\end{array}
$$

where col means column,

$$
\zeta=\frac{\lambda-\frac{i}{2}}{\lambda+\frac{i}{2}}, \quad \frac{i}{2}-\lambda=\frac{i \zeta}{\zeta-1}, \quad-\frac{i}{2}-\lambda=\frac{i}{\zeta-1} .
$$

Notice that we have

$$
\Phi_{1}^{*} T \Phi_{1}=\Phi_{1}^{*} X=X^{*} \Phi_{1}
$$

which implies the first equality in (5.7). Multiply both sides of (5.10) by $\lambda^{2}+\frac{1}{4}$ and use (5.11), (5.12) and the first equality in (5.7) to rewrite the result in the form

$$
\begin{aligned}
& \frac{i}{\zeta-1}\left(\left[\begin{array}{llll}
(\zeta-1) I_{p} & \left(\zeta^{2}-1\right) I_{p} & \cdots & \left(\zeta^{n}-1\right) I_{p}
\end{array}\right] X\right. \\
& \left.+X^{*} \operatorname{col}\left[\begin{array}{llll}
0 & \zeta^{-1}(\zeta-1) I_{p} & \cdots & \zeta^{-n}\left(\zeta^{n}-1\right) I_{p}
\end{array}\right]\right) \\
& =i\left[\begin{array}{lllll}
I_{p} & \zeta I_{p} & \cdots & \zeta^{n} I_{p}
\end{array}\right] \widehat{Q} \operatorname{col}\left[\begin{array}{llll}
I_{p} & \zeta^{-1} I_{p} & \cdots & \zeta^{-n} I_{p}
\end{array}\right] .
\end{aligned}
$$

The equalities for the coefficients corresponding to the same degrees of $\zeta$ on the lefthand side and on the right-hand side of (5.14) imply the second and the third relations in $(5.7)$. 
6. Factorization and similarity conditions. The block matrix

$$
K=\left[\begin{array}{c}
K_{0} \\
K_{1} \\
\vdots \\
K_{n}
\end{array}\right]
$$

where $K_{j}$ are $p \times(n+1) p$ matrices of the form

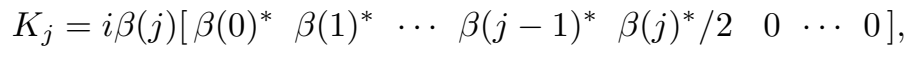

plays an essential role in [18]. From the proof of Theorem 3.4 in [18] the following result is immediate.

Proposition 6.1. Let a $(n+1) p \times(n+1) p$ matrix $K$ be given by formulas (6.1) and (6.2), and let conditions (3.13) hold. Then $K$ is similar to A:

$$
K=V_{-} A V_{-}^{-1}
$$

where $V_{-}^{ \pm 1}$ are block lower triangular matrices.

Proposition 6.1 is a discrete analog of the theorem on similarity to the integration operator [19].

REMARK 6.2. Note that $V_{-}^{-1}$ can be chosen so that

$$
V_{-}^{-1}\left[\begin{array}{c}
\beta_{1}(0) \\
\vdots \\
\beta_{1}(n)
\end{array}\right]=\Phi_{1} .
$$

Moreover, $V_{-}^{-1}$ is a factor of $S$, i.e., $S=V_{-}^{-1}\left(V_{-}^{*}\right)^{-1} \in \Omega_{n}$. Any matrix $S \in \Omega_{n}$ can be obtained in this way.

An analogue of Proposition 6.1 for the self-adjoint discrete Dirac system and block Toeplitz matrices $S$ follows from the proof of Theorem 5.2 in [11].

Proposition 6.3. Let a $(n+1) p \times(n+1) p$ matrix $K$ be given by formulas (6.1) and

$$
K_{j}=i \beta(j) J\left[\beta(0)^{*} \cdots \quad \beta(j-1)^{*} \quad \beta(j)^{*} / 2 \quad 0 \quad \cdots \quad c\right], \quad J=\left[\begin{array}{ccc}
0 & I_{p} \\
I_{p} & 0
\end{array}\right]
$$

where $\beta(k)$ are $p \times 2 p$ matrices. Let conditions $\beta(k) J \beta(k)^{*}=I_{p}(0 \leq k \leq n)$ hold. Then $K$ is similar to $A$ : $K=V_{-} A V_{-}^{-1}$, where $V_{-}^{ \pm 1}$ are block lower triangular matrices. Moreover, $V_{-}$can be chosen so that $S=V_{-}^{-1}\left(V_{-}^{*}\right)^{-1}$ is a block Toeplitz matrix. 
Acknowledgment. The authors are grateful to Professor W. Schempp and GALA project for the opportunity to meet in Grossbothen and discuss this paper.

\section{REFERENCES}

[1] D. Alpay and I. Gohberg. Connections between the Carathodory-Toeplitz and the Nehari extension problems: the discrete scalar case. Integral Equations Operator Theory, 37:125-142, 2000.

[2] Ph. Delsarte, Y. Genin, and Y. Kamp. Orthogonal polynomial matrices on the unit circles. IEEE Trans. Circuits and Systems, CAS-25:149-160, 1978.

[3] Ph. Delsarte, Y. Genin, and Y. Kamp. Schur parametrization of positive definite block-Toeplitz systems. SIAM J. Appl. Math., 36:34-46, 1979.

[4] V.K. Dubovoj, B. Fritzsche, and B. Kirstein. Matricial version of the classical Schur problem. Teubner-Texte zur Mathematik [Teubner Texts in Mathematics] 129, B. G. Teubner Verlagsgesellschaft mbH, Stuttgart, 1992.

[5] H. Dym. Hermitian block Toeplitz matrices, orthogonal polynomials, reproducing kernel Pontryagin spaces, interpolation and extension. Oper. Theory Adv. Appl., 34:79-135, 1988.

[6] B. Fritzsche and B. Kirstein. An extension problem for non-negative Hermitian block Toeplitz matrices. Math. Nachr., Part I, 130:121-135, 1987; Part II, 131:287-297, 1987; Part III, 135:319-341, 1988; Part IV, 143:329-354, 1989; Part V, 144:283-308, 1989.

[7] B. Fritzsche and B. Kirstein. On the Weyl matrix balls associated with nondegenerate matrixvalued Carathodory functions. Z. Anal. Anwendungen, 12:239-261, 1993.

[8] B. Fritzsche, B. Kirstein, and M. Mosch. On block completion problems for Arov-normalized $j_{q q^{-}} J_{q^{-}}$-elementary factors. Linear Algebra Appl., 346:273-291, 2002.

[9] B. Fritzsche, B. Kirstein, and K. Müller. An analysis of the block structure of certain subclasses of $j_{q q}$-inner functions. Z. Anal. Anwendungen, 17:459-478, 1998.

[10] B. Fritzsche, B. Kirstein, and A.L. Sakhnovich. Completion problems and scattering problems for Dirac type differential equations with singularities. J. Math. Anal. Appl., 317:510-525, 2006.

[11] B. Fritzsche, B. Kirstein, I. Roitberg, and A.L. Sakhnovich. Weyl matrix functions and inverse problems for discrete Dirac-type self-adjoint systems: explicit and general solutions. Operators and Matrices, 2:201-231, 2008.

[12] L. Golinskii and P. Nevai. Szegö difference equations, transfer matrices and orthogonal polynomials on the unit circle. Comm. Math. Phys., 223:223-259, 2001.

[13] G. Heinig and K. Rost. Algebraic methods for Toeplitz-like matrices and operators. Oper. Theory Adv. Appl., 13, Birkhäuser Verlag, Basel, 1984.

[14] M.A. Kaashoek and A.L. Sakhnovich. Discrete skew self-adjoint canonical system and the isotropic Heisenberg magnet model. J. Functional Anal., 228:207-233, 2005.

[15] A.L. Sakhnovich. A certain method of inverting Toeplitz matrices. Mat. Issled., 8:180-186, 1973.

[16] A.L. Sakhnovich. On the continuation of the block Toeplitz matrices. Functional Analysis (Uljanovsk), 14:116-127, 1980.

[17] A.L. Sakhnovich. Toeplitz matrices with an exponential growth of entries and the first Szegö limit theorem. J. Functional Anal., 171:449-482, 2000.

[18] A.L. Sakhnovich. Skew-self-adjoint discrete and continuous Dirac-type systems: inverse problems and Borg-Marchenko theorems. Inverse Problems, 22:2083-2101, 2006.

[19] L.A. Sakhnovich. Spectral analysis of Volterra's operators defined in the space of vectorfunctions $L_{m}^{2}(0, l)$. Ukr. Mat. Zh., 16:259-268, 1964.

[20] L.A. Sakhnovich. An integral equation with a kernel dependent on the difference of the arguments. Mat. Issled., 8:138-146, 1973. 
[21] L.A. Sakhnovich. On the factorization of the transfer matrix function. Sov. Math. Dokl., 17:203-207, 1976

[22] L.A. Sakhnovich. Integral equations with difference kernels on finite intervals. Oper. Theory Adv. Appl., 84, Birkhäuser Verlag, Basel, 1996.

[23] L.A. Sakhnovich. Interpolation theory and its applications. Mathematics and its Applications, 428, Kluwer Academic Publishers, Dordrecht, 1997.

[24] L.A. Sakhnovich. Spectral theory of canonical differential systems, method of operator identities. Oper. Theory Adv. Appl., 107, Birkhäuser Verlag, Basel, 1999.

[25] B. Simon. Orthogonal polynomials on the unit circle, Parts 1/2. Colloquium Publications, 51/54, American Mathematical Society, Providence, 2005.

[26] G. Teschl. Jacobi operators and completely integrable nonlinear lattices. Mathematical Surveys and Monographs, 72, American Mathematical Society, Providence, 2000. 